\title{
Mullerian Duct
}

National Cancer Institute

\section{Source}

National Cancer Institute. Mullerian Duct. NCI Thesaurus. Code C13260.

A pair of embryonic tubular structures that extend along the genital ridge and continue toward the cloaca, which in females will develop into the vagina, uterus, and fallopian tubes, and which will degenerate and be resorbed in males. 